\title{
Đî̀̂ NGHỊ MộT PHƯƠNG PHÁP XÂY DỰNG PHẦN MỀM DẠY HỌC
}

\author{
ĐỖ VĂN THÀNH, NGÔ ÁNH TUYẾT
}

\begin{abstract}
Constructing Tutoring System on Computer Network is a problem that is being interested by researchers. The main of this paper is to introduce a method for constructing a Tutoring System via presenting one for concrete problem of geometry. The Tutoring System describes approximately process of studying and teaching in class. The paper proposes a method for presenting pupil actions in a defined studying session. This presentation method permits to diagnostic knowledge status of pupils and to help them in any time in the studying session.
\end{abstract}

The experiment Tutoring System confirms possibility of distance education automatically controlled by computers.

\section{ĐẶT VẤN Đ产}

Việc xây dựng phần mềm dạy học luôn luôn là vấn đề thời sự được các nhà tin học và các nhà giáo dục quan tâm. Đến nay đã có nhiều phần mềm về chư đề này, nhưng một phần mềm mang lại hứng thú và hiệu quả thực sự trong quá trình dẫn dắt học sinh học tập thì còn khá hiếm. Một trong những bước tiến đáng kể trong lĩnh vực này là Cabri-géomètre ([1]) (CG), một phần mềm dạy học có nhiều đặc điểm khác biệt so với các phần mềm dạy học truyền thống, ở đây học sinh đóng vai trò hoàn toàn tích cực trong việc xây dựng kiến thức, thực chất đây là một vi thế giới cho phép người đọc tự khám phá thế giới hình học sơ cấp.

Tuy vậy, mặc dù không phủ nhận ích lợi cưa việc tự học, tự khám phá, các nghiên cứu gần đây chứng tó rằng việc học sẽ tốt hơn, nhanh hơn rất nhiều nếu học sinh luôn có được sự dẫn dắt cưa thầy và người ta mong muốn xây dựng những phần mềm dạy học mô tả gần giống như quá trình học của trò với sự hướng dẫn của thầy ở trên lớp.

Mồt vài năm gần đây người ta đã nói đến loại phần mềm dạy học được coi là "Hệ dạy học thông minh (HDHTM, Intelligent Tutoring System - tiếng Ảnh hay Tuteur Intelligent - tiếng Pháp) ([2], [3]). Việc xây dựng những phần mềm như vậy là hết sức phức tạp và chưa có một phương pháp thống nhất, tuy nhiên nó đang được các nhà tin học và giáo dục ở các nước tiên tiến hết sức quan tâm và có được một số kết quả bước đầu. 
Mặt khác hiệu quả của máy tính sẽ tăng lên rất nhiều nếu chúng được nối thành mạng, đó là thực tế ở các nước có trình độ tin học phát triển và là xu thế tất yếu của tin học. Do vậy, một trong những hướng được quan tâm là xây dựng những HDHTM trên mạng máy vi tính.

Bài này gồm 3 phần. Phần thứ nhất trình bày ngắn gọn về một số ý tưởng xây dựng phần mềm kiểu này đã và đang được nghiên cứu trong phòng thí nghiệm "Công nghệ dạy học và kỹ thuật nhận thức" của Viện nghiên cứu Tin học và Toán ứng dụng (LSD2-Imag) Grenoble - Cộng hòa pháp. Phần thứ hai trình bày một số kết quả nghiên cứu mà chúng tôi đã thực hiện trong chính phòng thí nghiệm trên. Phần cuối cùng là một vài suy nghĩ xung quanh việc xây dựng phần mềm dạy học theo hướng này.

\section{I - HDHTM TRONG VI THẾ GIÓI CABRI-GÉOMÈTRE}

Việc xây dựng HDHTM trong vi thế giới Cabri-géomètre là một trong những hướng nghiên cứu chính của chương trình nghiên cứu quốc qia: "Đề án Cabrigeomètre" (CG) thuộc Trung tâm nghiên cứu khoa học quốc gia Cộng hòa Pháp. Đó là vấn đề khá mới mé và có thể nói Phòng thí nghiệm LSD2-Imag là một trong những cơ sở khoa học tiên phong trên thế giới về vấn đề này. Nó liên quan chặt chẽ với hướng nghiên cứu: Mô hình hóa quá trình nhận thức của học sinh và những quy tắc để đề ra những quyết định sư phạm trong quá trình dạy học bằng máy tính. Những HDHTM này có nhiệm vụ dẫn dắt học sinh học môn toán nói chung và hình học nói riêng một cách tự động trong một vi thế giới mà ở đây là phần mềm CG.

Thử nghiệm đầu tiên là Hypercarre ([5]), ở đây người ta đặt ra một bài toán dựng hình hình học. Học sinh giải quyếtbài toán này trong môi trường CG. Sau khi học sinh đã dựng hình xong hệ thống phải phân tích hình vẽ để trả lời phép dựng ấy là đúng hay sai, đồng thời đưa ra những lời giải thích, giúp đỡ. Yêu cầu đặt ra là những trợ giúp này không được chung chung mà phải phù hợp với nhận thức ở thời điểm hiện tại của từng học sinh, muốn vậy người ta cần phải biết tất cá các trạng thái nhận thức của học sinh trong quá trình giải quyết vấn đề đã cho, mô hình hóa chúng, có cơ chế để dự đoán ở từng thời điểm học sinh thuộc loại nào (dựa trên quá trình làm việc của học sinh với hệ thống từ lúc bắt đầu).

Trong Hypercarre sử dụng Mô hình học sinh dưới dạng một vectơ, các trường của vectơ này chứa thông tin về trình độ hiện tại của học sinh, giá trị của chúng thay đổi trong quá trình học sinh làm việc trong hệ thống. Việc thay đổi này tuân theo các luật chuẩn đoán nhất định. Các lời giải thích hợp trình độ được đưa ra dựa trên mô hình học sinh này.

HDH thứ hai đã và đang được xây dựng lại được tổ chức theo một cách khác so với $\mathrm{HDH}$ trên, có sử dụng công nghệ ghép nối mạng. 
S. Tahri, C. Dubois, N. Balacheff, J. M. Laborde đề nghị xây dựng HDH theo mô hình sau: Tồ chức hai loai tram làm việc, một dành cho thầy giáo, một dành cho học sinh. Hai trạm đó (ở đây thực chất là các máy tính) cách xa nhau và được nối với nhau. Trên trạm làm việc của học sinh đã sẵn có phần mềm $C G$ và một cửa sổ giao diện, người học có nhiệm vụ giải quyết một bài toán hình học nào đó. Trên trạm làm việc của người thầy giáo luôn luôn có hình ảnh (trong thời gian thực) màn hình máy học trò. Thày giáo có thể theo dõi dần dần từng bước một mọi cách ứng xứ của học sinh trước vấn đề đã cho. Trên trạm này có một sơ đồ để thầy giáo có thể đánh dấu tất cà các cách ứng xử của học sinh, dựa vào đó HDH sẽ phân tích các hành động được đánh dấu để chẩn đoán tình trạng cưa học sinh, khả năng của họ, dẫn dăt họ một cách hiệu quả ngay trong quá trình làm việc.

HDHTM được xây dựng theo kiểu này gọi là HDHTM lai (tuteur intelligent hybride) bới nó gồm một gia sư nhân tạo (tuteur artificiel) và một gia sư người (Tuteur humain).

Trong cài đặt thể nghiệm cho HDH thứ hai này người ta đặt ra cho học sinh bài toán dựng hình sau: "Dựng đối xứng cưa một đoạn thẳng qua một đường thẳng". Các tác giả đã đưa ra 4 biến sư phạm (variables didactiques) như sau:

- phroơng của trục (nhận các giá trị: Ngang, Thẳng đứng, Xiên),

- phương của đoạn thăng (các giá trị: Ngang, Thẳng đứng, Xiên),

- giao của trục và đoạn thăng (các giá trị: Cắt nhau, Không căt nhau, Một mút của đoạn thẳng nằm trên trục),

- góc giũ̃a trục và doạn thăng (các giá trị: $0^{\circ}, 90^{\circ}$, góc khác).

Dựa trên các biến sư phạm này người ta đã phân ra 30 trường hợp [4], coi là tất cà các trường hợp có thể có trong tương giao giữa đọạn thẳng và đường thẳng đã cho trong bài toán. Vấn đề đặt ra là làm thế nào để hệ thống có thể kiểm soát và đánh giá được mọi phép dựng (đúng hay sai) của học sinh trong quá trình học sinh làm việc.

Đã có 2 cách tiếp cận được sử dụng trong việc xử lí vấn đề này.

Cách thứ nhất: (Đã được thể hiện một phần bằng chương trình và là nội dung chính trong luận án của $S$. Tarhi và $C$. Dubois được bào vệ tại LSD2-IMAG tháng 9 - 1993). Dựa trên sự thay đổi giá trị cưa các biến sư phạm [4], các tác giả nghiên cứu các phàn ứng cưa học trò và đề xuất một cách biểu diễn các lỗi cưa học sinh theo sự thay đổi này, họ mong muốn tìm một quy luật cho các lỗi. Tuy nhiên số lỗi được đề nghị đưa ra xem xét còn rất hạn chế do sự tăng nhanh của tổ hợp các phép dựng khác nhau, ngay cà các phép dựng đúng cưa học sinh cũng chưa được xét đầy đủ. Tương tự như Hypercarre, chỉ sau khi học sinh kết thúc các phép dựng, $\mathrm{HDH}$ mới phân tích và đánh giá kết quả. Như vậy tính tức thời chưa được quan tâm (xem [6]). Nhìn chung cách tiếp cận này chưa thóa mãn yêu cầu đặt ra vì thực sự việc hình thành các quy tăc để chuẩn đoán tự động tình 
trạng của học trò là một công việc khó khăn, nhất là việc hình thành các quy tắc để chuẩn đoán các lỗi.

Cách thứ hai: Cũng trong thời gian này N. Balacheff và $M$. Giry [7] đã sứ dụng lý thuyết đồ thị với việc nhúng đồ thị các phép dựng cưa học sinh trong đồ thị các phép dựng của thầy giáo (được gọi là đồ thị suy luận) bằng việc phân tích các phép dựng chuân từ các kết quả đi lên, và áp dụng phương pháp lập luận định tính (raisonnement explicatif) là phương pháp đã được dùng để biểu diển tri thức khi xây dựng $\mathrm{CG}$ để hình thành quy tắc chuẩn đoán tự động. Phương pháp này đã quan tâm đến tính tức thời của HDHTM nhưng chưa quan tâm đến thứ tự của phép dựng. Các phép dựng đúng cưa học sinh được hệ thống xem xét đã được tăng lên đáng kể so với cách tiếp cận thứ nhất, nhưng so với thực tế vẫn còn là ít và cũng chưa hình thành được cách biểu diển lổi, chưa được thể hiện dưới dạng chương trình.

Cả hai cách tiếp cận trên đều hướng tới việc xây dựng quy tắc chẩn đoán tự động tình trạng kiến thức của học sinh (kể cả các lỗi) bằng các luật, sau đó dùng kỹ thuật của việc xây dựng các hệ chuyên gia để xây dựng HDHTM (bằng việc xây dựng các mô tơ suy luận). Chúng tôi cho rằng với các bài toán chứng minh định lý điều này có thể khả thi do tính cấu trúc của lời giải bài toán, còn với các bài toán dựng hình thì phương pháp này chưa hằn phù hợp, vì thế dưới đây chúng tôi đề xuất việc biểu diễn tri thức bằng cách khác.

\section{II - BIÊU DIỄN TRI THƯC BĂNG MẠNG NGŨ NGHĨA}

Trước hết, theo chúng tôi những phẩm chất mà HDHTM cần phải có là $[6,8]:$

1. HDHTM phải có khả năng của người chuyên gia, cự thể:

+ Phải có khả năng đánh giá tất cả (hoặc phần lớn) các tình huống giải quyết đúng của học sinh trong quá trình tìm lời giải bài toán đặt ra cho họ.

+ Ở bất kỳ thời điểm nào cũng có thể đánh giá được công việc của học sinh, chỉ ra hành động mà học sinh vừa thực hiện là đúng hay sai, và trong từng ngữ cánh cụ thể thì hành động đúng tiếp theo là gì.

2. HDHTM phải có khả năng của người thầy giáo, thể hiện:

+ Phải có khà năng dẫn dắt học trò giải quyết vấn đề đã cho, cụ thể là:

- Bất kỳ lúc nào cũng có thể phân tích các hành động sai mà học sinh mắc phải, nguyên nhân, mức độ của sai lầm ấy.

- Trên cơ sớ này có chiến lược giúp học sinh vượt qua những hạn chế về tri thức của họ bằng việc chỉ ra những khái niệm, kiến thức phù hợp với từng học 
sinh trong hoàn cảnh cụ thể của họ, giúp họ tự vượt qua khó khăn hiện tại.

Theo chúng tôi khó khăn nhất là làm cho HDHTM có được phẩm chất thứ hai, bởi vì cho tới thời điểm hiện nay khoa học nhận thức còn chưa cho chúng ta biết một cách đầy đủ và định lượng về quá trình học, tình trạng tri thức của học sinh ờ mỗi thời điểm là rất phong phú, các bước đúng trong giải quyết vấn đề có thể là hữu hạ, nhưng các bước sai lại dường như có vé là vô hạn. Tuy nhiên bằng các thí nghiệm với học sinh, bằng phân tích tiên nghiệm và bằng kinh nghiệm của nguời thầy giáo thì có thể thấy rằng trong khi giải quyết một vấn đề cụ thể nào đó học sinh thường chỉ mắc phải một số lối chung. HDHTM cần phải xét đến ít nhất các lỗi chung này.

Chúng tôi coi rằng khả năng cưa HDHTM không chỉ thể hiện ở các phẩm chất nói trên mà còn được thể hiện ở khía cạnh tương quan so sánh giữa phần máy và phần người thầy. Cụ thể, trong HDHTM, vai trò của gia sư nhân tạo càng mạnh càng tốt, và ngược lại vai trò của gia sư người càng yếu càng tốt.

Quay lại bài toán dựng hình trên, đễ xây dựng HDHTM về vấn đề này chúng tôi đã tiến hành theo các bước sau:

a) Phân loại vấn đề đã cho.

b) Tìm tất cá các phép dựng hình đúng có thể có, cũng như sơ bộ các phép dựng hình sai. Sau đó phân tích các hành động này để rút ra những hành động mà học sinh sẽ sử dụng trong quá trình giải quyết công việc của họ.

c) Bằng cách dựa vào các phân tích kể trên, xây dựng mạng ngữ nghĩa cho vấn đề đặt ra.

d) Dựa vào mạng ngữ nghĩa đễ xây dựng chương trình.

Chúng tôi trình bày chi tiết hơn cách thực hiện các bước này.

\section{Phân loại các vấn đề}

Trong 30 vấn đề được đê nghị [4], có thể thấy rằng có nhiều vấn đề được giải quyết theo những cách khá giống nhau, chằng hạn các vấn đề: $29,21,27,15,18$ hoặc các vấn đề: $30,22,18,16,7$ là như vậy. Điều này đòi hỏi sự phân loại các vấn đề. Chúng tôi đã phân loại các vấn đề theo các biến sư phạm đã được đề nghị với thứ tư là:

- giao của trục và đoạn thẳng,

- góc giữa trục và đoạn thăng,

- hướng của trục,

- hướng của đoạn thănng,

theo đó nhận được một sự phân lớp. Theo sự phân lớp này hai nhóm I, II là quan trọng nhất, và nhóm I, VI hoặc II, VII có các tình huống dựng hình khá giống nhau [6]. Đó là những gợi ý ban đầu quan trọng để tổ chức xây dựng chương trình. 


\section{Tìm các phép dựng hình đúng và sai thường gặp}

Đế giải quyết vấn đề này, chúng tôi xuất phát từ định nghĩa đối xứng của một điểm qua một đường thẳng được cho bởi $\mathrm{D}$. Grenier (xem [9]):

Ký hiệu $A^{\prime} B^{\prime}$ là đối xứng của $A B$ qua đường thẳng $d$ với $A^{\prime}, B^{\prime}$ tương ứng là các điểm đối xứng của $A, B$ qua $d$. Khi đó 3 mệnh đề sau là tương đương:

1) $A^{\prime} B^{\prime}$ là đối xứng qua $d$ của đoạn thẳng $A B$.

2) $d$ là trung trực của các đoạn $A A^{\prime}$ và $B B^{\prime}$.

3) Các đoạn thẳng $A A^{\prime}, B B^{\prime}$ vuông góc với $d$ và cắt $\mathrm{d}$ tại điểm giữa của chúng.

Thực chất của việc tìm đối xứng của một đoạn thằng quy về việc tìm đối xứng cúa một điềm, theo 3 ) tức là giải quyết hai vấn đề chính sau:

i) Điểm đối xứng thuộc đường thẳng vuông góc với trục.

ii) Giao điểm của trục và đường thẳng vuông góc này là điểm giữa của hai điểm đối xứng nhau qua trục.

Người ta chỉ có thể giải quyết vấn đề ii) sau hoặc đồng thời với i). Theo hướng này ta có thể tìm được tất cả các thủ tục dựng đúng về mặt toán học, và phát hiện sơ bộ một số sai lầm mà học sinh thường gặp phải.

Sự phức tạp ở đây là chỗ: Mỗi một thư tục dựng đúng về mặt toán học sẽ sán sinh ra nhiệu thư tục dựng hình đúng của $\mathrm{HDH}$, vì $\mathrm{HDH}$ quan tâm đến yếu tố thời gian thực. Tuy nhiên việc giải quyết toán học này cho chúng tôi nhận thấy rằng chỉ có một phép dựng (kể cả phép dựng đúng và phép dựng sai) được lặp đi lặp lại. Đó cũng là một trong các nguyên nhân để chúng tôi dùng mạng ngữ nghĩa biểu diễn tri thức cho vấn đề đã cho.

\section{Xây dựng mạng ngữ nghĩa}

Chúng tôi đã xây dựng mạng ngữ nghĩa cho từng nhóm vấn đề. Trong các mạng ngữ nghĩa này mỗi nhánh của mạng tương ứng với một thủ tục dựng hình đúng. Mỗi nút của mạng ứng với một tình trạng học sinh. Việc biểu diển này có một điểm lợi sau đây:

+ Lập trình và quản lý chương trình dễ dàng.

Theo mạng ngữ nghĩa của nhóm tương ứng, ta có thể lập trình để giải quyết vấn đề đã cho theo các nhóm, dựa vào mạng ta cũng có thể phát hiện lối do việc lập trình gây ra, và sửa chữa các lỗi đó một cách dễ dàng.

+ Biểu diễn được tất cả (hoặc phần lớn) các tình huống trong quá trình dựng hình của học sinh, đó là các phép dựng hình đúng và thậm chí cả các phép dựng lỗi. Thật vậy:

i) Ớ vị trí bất kỳ nào đó của mạng ngữ nghĩa ta đều có thể xác định được tất cả (hoặc phần lớn) các phép dựng đúng có thể có tiếp theo, và trong ngữ cảnh 
này ta có thể xác định được các lỗi chư yếu của học sinh (các lỗi này được liệt kê từ kinh nghiệm của ngưồi thầy và từ các kết quả của các thí nghiệm trên các nhóm học sinh).

ii) Một thực tế chắc chắn không tránh khỏi là ban đầu khi sử dụng $\mathrm{HDH}$ ta thấy ở một vị trí nào đó của mạng ngữ nghĩa cần phải bỗ sung hoặc loại bó một vài phép dựng lỗi nào đó chưa thích đáng, thậm chí cũng có thể cả đối với những phép dựng chuẩn. Rõ ràng mạng ngữ nghĩa rất phù hợp với công việc này.

+ Theo mạng ngữ nghĩa ta có thể giải quyết được vấn đề chẫn đoán tự động tình trạng của học sinh.

Với một tình trạng của học sinh (ứng với một nút của mạng ngữ nghĩa), ta có thể biết được những hành động nào là thích đáng, những hành động nào học sinh có thề sư dụng tiếp theo.

Theo thứ tự phân bậc của mạng ngữ nghĩa, ta tìm thấy cách đánh giá khá năng học sinh. Dựa theo các nhánh của mạng ta hoàn toàn biết được hành động của học sinh vừa thực hiện là đúng hay sai, dự đoán, đề ra cách khắc phục các lỗi đó. Tức thời ta có thể cho học sinh một sự giúp đỡ thích hợp khi có yểu cầu hoặc khi thầy giáo thấy cần thiết.

Nếu các lỗi của học sinh cách xa gốc của mạng, điều đó nói lên rằng học sinh này có khả năng giải quyết vấn đề đã cho, sự yếu kém về tri thức của anh ta không phải là trầm trọng, nhưng sẽ hoàn toàn khác nếu lỗi của anh ta gần gốc mang.

Theo vị trí của lỗi, ta cũng biết được nguồn gốc và mức độ của lỗi, trên cơ sở đó ta có thể cho học sinh những đánh giá thích hợp, những lời khuyên thích đáng, những khái niệm, kiến thức cũ quan trọng đối với tình trạng tri thức của học sinh. Thậm chí theo mạng ngữ nghĩa ta cũng biết được cánh giải của học sinh có tốt hay không. Một cách giải là tốt nếu số các nút từ gốc đến nút kết thúc của mạng là nhỏ theo một tiêu chuẩn nào đó.

+ Mạng ngữ nghĩa là cơ quan trọng để xây dựng sơ đồ.

Theo mạng ngữ nghĩa ta có thể thấy được tất cả các phép dựng mà học sinh có thể sử dụng trong quá trình dựng hình của họ. Một sơ đồ của một nhóm cần phải thỏa mãn một số yêu cầu sau:

a) Nó bao gồm tất cá các hành động có thể có của học sinh (trên sơ đồ mỗi hành động như vậy tương ứng với một hoặc tổ hợp hai button).

Các hành động này được mô tả phù hợp với các tùy chọn của $\mathrm{CG}$.

b) Các tỗ hợp hợp lý của các hành động này sẽ cho tất cả các tình huống có thể có trong một quá trình giải quyết vấn đề đặt ra cho học sinh. Vì thế không thể xây dựng sơ đồ chung cho cá 30 vấn đề như [4] đã làm bởi sơ đồ khi đó sẽ rất lớn và không thể thể hiện được các tình huống có thể xảy ra.

Việc xây dựng mạng ngữ nghĩa dựa trên các hành động rút ra từ các thủ tục 
dựng hình chuân, các phép dựng hình sai về toán học và trong môi trường CG. Trước hết chúng tôi xây dựng mạng ngữ nghĩa cho tất cả các thủ tục dựng hình chuẩn. Sau đó biểu diễn các lối mà học sinh thường gặp vào các nút của mạng ngữ nghĩa nói trên.

Chúng tôi đã đề nghị phân ra ba loại lỗi:

*) Lỗi loại 1: là một phép dựng đúng về mặt trực giác nhưng không được phép trong môi trường $\mathrm{CG}$ (Có thể biết được điều đó qua việc làm biến dạng hình vẽ hoặc sử dụng các tùy chọn để đo hay kiểm tra tính chất các hình trong $\mathrm{CG}$ từ version2. trở lên).

*) Lỗi loại 2: là một phép dựng có liên quan về phương hướng với các phép dựng trước, nhưng hoàn toàn không đúng.

*) Lỗi loại 3: là một phép dựng không liên quan gì đến phép dựng trước, với phép dựng ấy theo logic thông thường không thể hiểu vì sao học sinh lại làm như vậy.

Các khái niệm lỗi theo chúng tôi mới chì là tương đối, phụ thuộc nhiều vào kinh nghiệm sư phạm của người xây dựng $\mathrm{HDH}$ và có thể được tinh chỉnh hơn nữa.

Chúng tôi đã đề nghị phân loại 30 vấn đề thành 8 nhóm, nhưng có thể gộp vào thành 5 nhóm khac nhau, để xây dựng chương trình ta sẽ sử dụng 5 loại card khác nhau (trong mỗi loại có thể gồm nhiều card khác) ứng với 5 nhóm này [6].

Việc xây dựng mạng ngữ nghĩa cho các nhóm vấn đề khác được tiến hành tương tự, nhưng với các nhóm $\mathrm{I}$, VI thì việc này rất phức tạp. Ơ đây học sinh không những dựng hai điểm đối xứng bằng phương pháp giống nhau hoặc không (theo $\mathrm{S}$. Tarhi và $\mathrm{C}$. Dubois là độc lập hay không độc lập) mà chúng còn được tiến hành đồng thới hoặc không.

Ta có thể hình dung được sự phức tạp đó. Để dựng đối xứng của một điểm qua trục ta có 27 cách [6]. Mỗi một cách dựng đối xứng của điểm mút thứ nhất kết hợp với một cách dựng điểm đối xứng của điểm mút thứ hai sẽ cho ta một cách dựng đối xứng cưa đoạn thẳng qua trục, ta có $27 * 27$ cách. Nếu ta dựng đối xứng của đoạn thẳng theo cách sau: thực hiện lần lượt một hoặc một phần các phép đối xứng của điểm mút thứ nhất, sau đó cũnh như vậy với điểm mút thứ hai, ta lại có ít nhất là $27 \times 27$ cách nữa. Vậy thì ta có ít nhất là $2 \times 27 \times 27=1458$ cách. Điều này là quá phức tạp, đặc biệt khi chúng ta mong muốn xử lí các lỗi.

Để đối phó với độ phức tạp này, chúng tôi đã xây dựng mạng ngữ nghĩa của nhóm này theo cách sau đây: Phân tích thật tị mî tất cà các trạng thái khác nhau của các thư tục chuẩn trong toán học để tìm ra tất cá các thư tục dựng hình chuẩn trong CG. Ở đây ta không cần biết phép dựng nào là cho điểm mút thứ nhất, phép dựng nào là cho điểm mút thứ hai, tức là chỉ dựa vào các hành động đã được đề nghị khi tìm các thủ tục chuẩn trong toán học. Tuy nhiên theo phương pháp này ta cũng có nguy cơ đề nghị quá nhiều thư tục. Lúc này vai 
trò của người thầy giáo là rất quan trọng. Bằng kinh nghiệm sư phạm của mình người thầy có thể hạn chế bớt những thủ tục dựng hình rất hiếm khi xáy ra.

Với nhóm I, VI chúng tôi đã xây dựng mạng ngữ nghĩa với khoảng 150 thủ tục dựng hình chuần. Theo chúng tôi 150 thư tục này phản ánh khá đầy đủ những tình huống thường xẩy ra nhất khi học sinh giải quyết nhóm vấn đề này.

\section{Xây dựng chương trình}

a) Phương pháp xây dựng:

Ta nhận thấy rằng trong các mạng ngữ nghĩa của các nhóm có một số phép dựng chung. Vì thế khi lập trình ta sẽ sử dụng các hàm và các thủ tục chung, chúng sẽ được đặt trong chương trình (script) của CARD nếu chúng được sử dụng trong một nhóm vấn đề, hoặc trong chương trình của STACK nếu chúng là chung cho mọi nhóm vấn đề.

Việc lập trình được dựa vào mạng ngữ nghĩa. Ở đây khi xử lí các lỗi chúng tôi đề nghị một cách như sau: Nếu học sinh phạm phải lỗi loại $1, \mathrm{HDH}$ nhắc nhở họ kiếm tra lại lỗi đó, và gợi ý họ để họ có thể giải quyết tiếp bài toán. Nếu học sinh phạm lỗi loại $2, \mathrm{HDH}$ sẽ phân tích lỗi và nếu cần thiết có thể nêu tất cả hành động hợp lý tiếp theo để học sinh tự chọn. Nếu học sinh phạm lỗi loại $3 \mathrm{HDH}$ không những phải phân tích lỗi đó, mà còn phải trang bị kiến thức thêm cho họ, dẫn dắt họ giải quyết tiếp bài toán theo cách tốt nhất trong tình huống ấy.

Trong cài đặt cụ thể chúng tôi đã tổ chức 2 STACK, một stack mang tên "Principale" cho phép chọn bất kỳ một vấn đề nào theo giá trị của biến sư phạm, và Stack "Diagnostic" để chẩn đoán tình trạng học trò. Trong Stack thứ hai với một nhóm vấn đề có thể có một vài sơ đồ (nói chung là một). Một sơ đồ có thể xuất hiện nhiều lần vì cũng giống như một số ngôn ngữ lập trình khác, khi chương trình quá lớn, để hệ thống dịch được chương trình đó ta phải chia chương trình đó thành các phần nhỏ hơn.

\section{b) Đánh giá:}

Việc lập trình cho nhóm I, VI là phức tạp nhất. Có thể nói rằng chúng tôi đã xây dựng $\mathrm{HDH}$ có hai phầm chất kể trên, ở đó vai trò của gia sư người chỉ là theo dõi và đánh dấu cho đúng các hành động mà học sinh đã thực hiện, và nhấn vào một button cố định khi cần đánh giá học sinh hoặc học sinh cần sự giúp đỡ Theo chúng tôi có thể trong một tình trạng cụ thể nào đó của học sinh (nút của mạng ngữ nghĩa) còn có những phép dựng sai chưa thích đáng đã được đề nghị, nhưng đưa vào mạng ngữ nghĩa ta sửa chữa không khó khăn lắm những nhược điểm đó. Chúng tôi muốn nhấn mạnh rằng để xây dựng được những HDHTM bên cạnh kiến thức tin học rất cần kinh nghiệm của người thầy giáo, các kinh nghiệm này có thể được tích lũy và đưa vào hệ dần dần. 


\section{III - CÁC KẾT LUẬN}

Ý tưởng tổ chức hai kiểu trạm làm việc, một cho thầy, một cho học trò là một ý tưởng sư phạm rất hay, tạo ra khả năng học sinh tự học, tự khám phá thế giới bài học, môn học có sự dẫn dắt của người thầy. Nó mở ra triển vọng tốt đẹp của giáo dục từ xa bằng máy tính. Các HDHTM xây dựng theo kiểu này mới chì được nghiên cứu thể nghiệm trên vi thế giới $\mathrm{CG}$, theo ý tưởng này người ta có thể xây dựng các HDHTM trên các vi thế giới khác về Đại số, Lượng giác,... Một phần mềm dạy học như vậy không chỉ là một chuyên gia mà còn là một thầy giáo. Người ta đã nghiên cứu nhiều và đã hình thành khá rõ ràng đường lối chung để xây dựng một hệ chuyên gia, nhưng về thầy giáo còn rất ít. Chúng tôi cho rằng xây dựng sao cho $\mathrm{HDH}$ có được vai trò của người thầy giáo là khó khăn hơn nhiều bởi lẽ ta cần phải xử lí những sai lầm về tri thức của học trò.

Để biểu diễn tri thức, trong trí tuệ nhân tạo sử dụng ba cách:

Trước tiên phải kể đến việc dùng ngôn ngữ logic và biểu diễn tri thức dưới dạng các sự kiện (fact) và các luật (rule), cách biểu diễn này rất gọn và đẹp đẽ, nhất là đã có rất nhiều các phương pháp lập luận trên cơ sở tri thức như vậy. Tuy nhiên cách này không có cấu trúc và chỉ dựa vào nó sê rất khó thấy được tất cả các thông tin có liên quan đến một đối tượng nào đó và quan hệ giữa các đối tượng ấy, trong phần mềm dạy học các vấn đề đó càng trở nên phức tạp (do cần phải xử lí lỗi).

Cách thứ hai biểu diễn tri thức dưới dạng đồ thị các đối tượng. Cách này không phù hợp với vấn đề đặt ra.

Cách thứ ba là biểu diễn tri thức dưới dạng mạng ngữ nghĩa, cách biểu diễn này thường rất cồng kềnh nhưng khắc phục được những hạn chế trên. Một mang ngữ nghĩa thông thường là sự kết hợp đan chéo của 3 loại đồ thị: đồ thị suy luận, đồ thị các khái niệm và đồ thị các quan hệ. Một mạng ngữ nghĩa như vậy là hoàn toàn tương đương với cách biểu diễn tri thức dưới dạng các luật. Với vấn đề đặt ra thực chất chúng tôi chỉ sử dụng đồ thị suy luận. N. Balacheff và $\mathrm{M}$. Giry cũng đặt vấn đề theo hướng này, nhưng cách giải quyết cụ thể thì hoàn toàn khác [7].

HDHTM là sự kết hợp của trí tụe nhân tạo, công nghệ lập trình, khoa học nhận thức và tâm lí, khoa học sư phạm, và đương nhiên, các tri thức khoa học khác có liên quan. Đã có những nhóm (thuộc đề án $\mathrm{CG}$ ) thử nghiên cứu dùng ngôn ngữ Prolog để xây dựng HDHTM, mặc dù Prolog là ngôn ngữ rất thích hợp cho các vấn đề trí tụê nhân tạo, nhưng không phải là môi trường thuận lợi để chuyển vận sư phạm trong quá trình học của trò, dạy của thầy. Chính vì lẽ đó cho đến nay ngôn ngữ lập trình hướng đối tượng Hypertalk tỏ ra là thích hợp nhất cho việc xây dựng phần mềm dạy học thông minh theo kiểu tổ chức hai trạm làm việc nói trên. Nhất là ngôn ngữ Hypertalk có khả năng nối được với các ngôn ngữ $\mathrm{Pascal}$ và $\mathrm{C}$. Điều này mở ra triển vọng là sẽ sử dụng các ngôn ngữ 
C, Pascal để giải quyết nhiệm vụ người chuyên gia của HDHTM, còn ngôn ngữ Hypertalk giải quyết nhiệm vụ cưa người thầy giáo (đặc biệt ở khía cạnh giao diện trò-máy-thầy).

\section{TÀI LIÊU THAM KHẢO}

1. Baulac Y., Laborde J. M., Cabri-Géomètre: Un logicel pour un nouvel apprentissage de la géomètrie, Actes du Congrés Educcation et Informatique, Paris: UNESCO, 1989, Vol. 2, 467-472.

2. Laborde J.M., Systèmes Tutoriels Intelligents et interactions éducatives, Quatrièmes Assises de l'intelligence artificielle et de la formation, Paris, 18-19 Juin, 1990 (Publication sollcitée par "Revue de la formation continue et de l'EAO).

3. Laborde J.M., Dessigning Intelligent Tutorial Systems: The case of geomtry and Cabri-Geomètre, IFIP WG 3.1, Working Conference on Educational Software at the Secondary Education Level, Reykjavik, 1989.

4. Tarhi S., Modelisation de l'interaction didactique: un tuteur hybride sur Cabri-géomètre pour l'analyse de decsitions didactiques, Thèse Grenoble: Universiré Joseph Fourier et Lsd2-Imag-CNRS, France, 1993.

5. Capponi B., hypercarre, Problemes d'un systeme tutoriel en Géomètre, in Nicaud J. F. et Baron M. (eds), Actes des II èmes journées d'EIAO, Cachan, 1991, 77 - 88.

6. Do Van Thanh, Rapport de fin du stage, LSD2-CNRS, France, 1993.

7. Balacheff N., Giry M., Diagnodtic et raisonnement explicatif dans Cabri-géomètre, in Brezillon P. et Karsenty L. (eds), Explication et coopération homme-machine: Définition d'un nouveau cadre pour la génération d'explication, Paris: CNAM, 1993.

8. Do Van Thanh et Ngo Anh Tuyet, Propossition d'une methode de construction d'un Tuteur Intellgent, Actes du Premiere colloque regional des Pays Fancophones du sud-est Asiatique, 20-24, Janvier, 1995 à Ho Chi Minh ville, Didactiques des Disciplinnes Scientifiques et Formation des Enseignants: Présentation d'outils informatiques.

9. Grenier D., Construction et Etude du fonctionmement d'un processus d'enseignement sur la symétrie orthoronale en sixième, Thèse Grenoble: Université Joseph Fourier, France, 1988.

Bộ môn Tin học

Truòng DHSP Hd Nội 1.

Nhận bài ngày 24-4-1995 